\title{
D'une réforme à l'autre : Le droit tutélaire à l'épreuve de son application
}

Benoît Eyraud*

Dans un contexte législatif et médiatique chargé, la loi réformant la protection juridique des majeurs est définitivement adoptée le 5 mars 2007. La présentation du droit des incapacités est profondément remaniée. La présomption de capacité, qui constitue depuis la naissance du Code civil la modalité fondamentale du principe d'égalité civile, inaugure toujours le Livre 11 :

"La majorité est fixée à dix-huit ans accomplis ; à cet âge, chacun est capable d'exercer les droits dont il a la jouissance ${ }^{1}$. »

Mais l'exception à cette présomption, qui est la pierre angulaire du droit des incapacités, est entièrement reformulée ${ }^{2}$ :

«Les personnes majeures reçoivent la protection de leur personne et de leurs biens que leur état ou leur situation rend nécessaire selon les modalités prévues au présent titre.

Cette protection est instaurée et assurée dans le respect des libertés individuelles, des droits fondamentaux et de la dignité de la personne. Elle a pour finalité l'intérêt de la personne protégée. Elle favorise, dans la mesure du possible, l'autonomie de celle-ci ${ }^{3} . »$

\footnotetext{
* Sociologue au Centre d'étude et de recherche sur les pratiques de l'espace, Lyon.

1. Article 414 du Code civil.

2. L'article 488 du Code civil « 68 » formulait à partir de son deuxième alinéa : «- Est néanmoins protégé par la loi, soit à l'occasion d'un acte particulier, soit d'une manière continue, le majeur qu'une altération de ses facultés personnelles met dans l'impossibilité de pourvoir seul à ses intérêts. - Peut pareillement être protégé le majeur qui, par sa prodigalité, son intempérance ou son oisiveté, s'expose à tomber dans le besoin ou compromet l'exécution de ses obligations familiales. »

3. Article 415 du Code civil.
} 
Cette reformulation insiste sur les principes de la protection organisée par ce droit (respect des libertés individuelles, droits fondamentaux, dignité, autonomie) plutôt que sur les incapacités auxquelles il s'agit de remédier. Elle ancre la réforme dans son époque ${ }^{4}$ en accordant une attention particulière «à la personne » à qui ce droit s'adresse en cherchant notamment à éviter la stigmatisation. Elle constitue une actualisation dans le droit tutélaire de "l'opposition au moins bicentenaire entre un principe de liberté proclamé et la volonté de secourir ou de surveiller les plus démunis, souvent contre leur gré..$»$

Cette contribution entend mettre en perspective cette opposition entre la présomption de capacité et l'exception de la protection en décrivant comment les pouvoirs donnés aux différents acteurs qui l'appliquent varient en fonction des évolutions de la politique psychiatrique, de celles de l'État social ou encore de la figuration civile de la personne. À travers ces évolutions, c'est l'équilibre entre les principes de liberté et de protection qui est étudié dans une perspective historique, à partir de la fin de la prise en charge asilaire globale des incapacités que la loi du 3 janvier 1968 entérine.

Les enjeux de déspécification de la prise en charge des incapacités au moment de la réforme de 1968 sont présentés dans un premier temps ; la mise en œuvre pratique de cette réforme est ensuite décrite pour souligner comment la souplesse du dispositif tutélaire a permis son adaptation à des publics variés ; dans un troisième temps, quelques axes de la récente réforme du 5 mars 2007 sont examinés pour souligner les tensions entre ce qui relève d'un droit civil commun et de droits sociaux plus spécifiques.

\section{LA REFORME DE 1968 : UNE RECOMPOSITION DES POUVOIRS JUDICIAIRE ET PSYCHIATRIQUE}

Au milieu des années soixante, les régimes d'incapacité de droit commun sont désuets depuis longtemps. Le régime asilaire d'exception s'est de fait imposé ${ }^{6}$ tout en faisant l'objet de critiques

4. Tout au long des années 1990 et 2000 , de nombreux États ont révisé leur législation tutélaire. Ce mouvement a été encouragé par les recommandations du Conseil de l'Europe et a conduit à la mise en place d'une convention entre États signée à La Haye le 13 janvier 2000 sur la protection internationale des adultes. Cf. Recommandation $n^{\circ} \mathrm{R}(99) 4$ du Conseil de l'Europe sur les principes concernant la protection juridique des majeurs incapables, adoptée par le Comité des ministres, le 23 février 1999.

5. Thierry FOSSIER, Michèle HARICHAUX, «La tutelle à la personne des incapables majeurs : l'exemple du consentement à l'acte médical », Revue de droit sanitaire et social, $\mathrm{n}^{\circ} 1,1991$, p. 1.

6. Loi sur les aliénés n 7443 du 30 juin 1838, art. 31 à 38. Dans le code Napoléon, la mise sous tutelle des biens résultait du régime de l'interdiction, la mesure d'interdiction 
importantes ${ }^{7}$, critiques qui conduiront à une transformation progressive en profondeur de la psychiatrie publique ${ }^{8}$, dont l'acte inaugural est pour beaucoup la fameuse circulaire de 1960 organisant sur un mode sectorisé la politique psychiatrique. La réforme du 3 janvier 1968 survient ainsi alors que les pratiques évoluent et qu'un cadre légal nouveau devient de plus en plus nécessaire.

\section{La dissociation de la protection des biens de celle de la personne}

L'axe central de la loi du 3 janvier 1968 est la dissociation de l'organisation de la protection des biens de celle du soin psychiatrique. Cette dissociation a été rendue obligatoire d'abord pour des raisons techniques. La création, pendant l'entre-deux-guerres, de services «libres » ou « ouverts » ne relevant pas des procédures administratives de la loi de 1838 et dédiés aux malades qui acceptent les soins, a exclu ceux-ci des dispositifs de protection des biens prévus par cette loi. De même, les malades non internés ne bénéficiaient pas du système de l'administration provisoire, ce qui a fait dire à Henri Ey que la loi de 1838 était celle du «tout ou rien ${ }^{9} »$. À ce défaut majeur s'ajoutent alors de nombreuses autres difficultés. À l'intérieur même de l'hôpital, l'administration provisoire devient de plus en plus problématique, la réduction des temps de séjour, la multiplication des hospitalisations de courte durée, et la complexification des biens des internés (en raison de la sécurité sociale, la retraite, la prévoyance...) rendant difficile le travail d'administrateurs peu formés.

Ces questions sont posées à partir de 1963 dans le cadre d'une « commission de réforme de la législation sur les aliénés » participant à la révision au long cours du Code civil menée sous la houlette de Jean Carbonnier.

La commission, composée de juristes et de psychiatres, s'accorde sur une décision lourde de conséquences, à savoir la dissociation de ce qui relève de l'administration des biens et de la liberté des personnes ${ }^{10}$.

étant alors déjà dissociée de la séquestration. Elle ne résolvait pas tous les problèmes posés par les insensés, dont «le destin social n'était alors pas complètement scellé ». Avec la loi de 1838 , l'interdiction juridique est disqualifiée au profit de l'expertise médicale et de l'internement. cf. Robert CASTEL, L'ordre psychiatrique, Paris, Ed. de Minuit, 1976, p. $24 ; 51-52 ; 169-174 ; 220$.

7. Les critiques de l'asile n'ont pratiquement jamais cessé depuis la promulgation de la loi de 1838

8. Nicolas HENCKÈS, Le nouveau monde de la psychiatrie française. Les psychiatres, l'État, et la réforme des hôpitaux psychiatriques de l'après-guerre aux années 70 . Thèse soutenue à l'EHESS en 2007.

9. Livre blanc de la psychiatrie française, Toulouse, Ed. Privat, t. I, 1965, t. 2, 1966, t. 3, 1967.

10. Bailly-Salin, à qui il revenait de présenter le projet de réforme à l'Évolution psychiatrique, indique ainsi en préambule que «la première ligne de force qui avait été déga- 
Personne ou presque n'a, semble-t-il, voulu discuter ce choix ${ }^{11}$, qui est présenté alors comme «une simple opportunité ${ }^{12}$ », et qui repose en partie sur des interprétations du droit. Dès 1952, dans un article de L'Information psychiatrique, les auteurs interprètent la doctrine juridique issue des cours de Colin et Capitant, en distinguant « une protection de la personne, objet du droit administratif », d'une «protection $d u$ patrimoine relevant du droit privé ${ }^{13}$. Ils en concluaient qu'il fallait attribuer exclusivement au pouvoir judiciaire la protection des biens, celle-ci étant «un domaine où la compétence de la Justice est évidente et incontestée ».

Du côté des juristes, l'enjeu est de poser une limite claire entre le domaine d'intervention du droit positif, à savoir les règles relatives à la gestion des biens, et ce qui ne relève pas du droit mais des relations interpersonnelles à l'intérieur de la famille. Quelques décennies plus tard, Thierry Fossier explique le refus de légiférer sur la protection de la personne, ce qu'il qualifie d'incohérence de la loi de 1968 :

«Jean Carbonnier s'en remettait à l'autorité parentale continuée au-delà de l'âge de la majorité, une cellule chaleureuse, une sorte de petit contrat familial dans lequel le malade abdique une bonne partie de la liberté en habitant chez ses parents, leur demandant l'autorisation pour un tas de choses, mais étant protégé en cas de coup dur... L'idée de Carbonnier était que ce n'était pas la peine de rentrer dans le détail si la personne n'avait pas envie d'être soignée... Et il n'avait nullement l'intention de détailler l'équipement d'une protection de la personne ${ }^{14}$. »

Du côté des psychiatres, cette position traduit le souci de préciser les règles communes de droit civil applicables aux majeurs incapables, quelle que soit l'origine de cette incapacité, et de permettre ainsi une déspécification du droit des malades mentaux. Pour les «psychiatres réformateurs », la volonté de dissocier la question de la protection des biens et celle de la personne tient précisément à leur préoccupation de

gée consistait à séparer radicalement les textes concernant l'administration des biens et les textes relatifs à la liberté des personnes », Livre blanc... op. cit., t.1, p.107.

11. Quelques voix discordantes sont toutefois repérables. La proposition de réforme d'Henri Baruk, de l'académie de médecine, se fonde par exemple sur le refus de cette dissociation : "Nous avons envisagé une législation à la fois pour la personne et pour les biens. Il est impossible de procéder autrement. » Les raisons évoquées sont relatives aux « données médicales ». Cf. Henri BARUK, « Projet de loi sur la révision de la loi du 30 juin », Annales médico-psychologiques, 1967, p. 752.

12. Jean CARBONNIER, préface à Jacques MASSIP, Les incapacités, Paris, Répertoire Defrénois, 2002, p. 23.

13. Xavier ABÉLY, Georges DAUMÉZON, Lucien BONNAFÉ, « Des dispositions à inclure dans le Code civil concernant les malades mentaux », L'Information Psychiatrique, $\mathrm{n}^{\circ} 4,1954$, p. 152.

14. Entretien avec Thierry Fossier. 
ne pas élaborer une nouvelle loi ségrégative. Faute de pouvoir abroger de manière générale la loi de 1838 au profit du droit commun, ils préfèrent ne pas mettre le doigt dans un engrenage qui risquerait de produire de nouveau une loi spécifique pour les malades mentaux. Ils sont donc très favorables à une loi s'intéressant uniquement à la question de la protection des biens et s'adressant à tout public, et non pas spécifiquement aux malades mentaux.

Aux yeux du juriste comme à ceux des psychiatres, il ne fait donc pas de doute que les règles de protection de la personne ne doivent pas être davantage précisées dans le droit commun. Cette éviction empêchera pendant longtemps que les débats relatifs au consentement au soin et plus largement aux actes personnels se posent à l'intérieur du droit commun. Les réflexions et les débats menés depuis longtemps dans le domaine de la psychiatrie, mais aussi de l'éthique médicale, n'ont rebondi que tardivement et sans être approfondis dans le champ du droit civil ${ }^{15}$.

\section{L'articulation équivoque de l'activité thérapeutique et de l'activité judiciaire}

La séparation de la protection de la personne et celle des biens s'est déclinée par l'affirmation, dans l'article 490-3 du Code civil, de l'autonomisation de la procédure de la protection des intérêts civils au regard de la démarche thérapeutique et de la création d'une nouvelle mesure, la sauvegarde de justice. La suppression de l'article 31 de la loi de 1838 prononçant la mise automatique sous administration provisoire lors de l'internement ouvre la voie à l'affirmation de la dissociation du lieu d'hébergement, des modalités thérapeutiques et des modalités de protection des intérêts civils ${ }^{16}$, et devient le symbole de la fin du régime asilaire $^{17}$, revendiqué aussi bien par les psychiatres que par les juristes :

«Ce cloisonnement des procédures constitue une garantie importante pour la liberté individuelle et pour l'individualisation des mesures de protection. ${ }^{18}$

15. Pendant longtemps, la question du consentement à l'acte médical ne se posait pas, ni pour le droit commun, ni pour le droit des incapables. La primauté étant accordée à la bienfaisance du médecin. Encore aujourd'hui, en cas d'urgence, il appartient au médecin, qui n'a alors besoin d'aucune autorisation, de donner les soins nécessaires. Cf. Article $42 \S .2 \mathrm{du}$ Code de déontologie médicale.

16. Article 490-1 du Code civil.

17. Castel formule quelques années après l'importance de cette rupture en remarquant que «l'aliénation mentale cesse alors d'être cette catégorisation massive, à la fois médicale, administrative et juridique qui annulait les unes par les autres toutes ces déterminations et les résumait dans un statut d'exception. » Robert CASTEL, La gestion des risques, Paris, Ed. de Minuit, 1981, p. 37.

18. Luc HARTEMANN, Droit civil : les personnes et les incapacités, Paris, L'Hermès, 2002, p. 128 
Mignot le formulait ainsi : "Il est capital, sur le plan thérapeutique, que ces malades, lorsqu'ils sont en état de s'occuper eux-mêmes de leurs affaires, aient la possibilité de le faire et ne soient pas tenus en lisière, car cette reprise de responsabilité est un élément très important de la restauration de leur personnalité. Dans ces conditions, le régime qui protège leurs biens doit être essentiellement mobile; il faut pouvoir facilement passer d'un régime de protection au droit commun, ou modifier l'importance de la tutelle exercée sur eux ${ }^{19}$. »

En conséquence, demande a été faite de prévoir trois régimes différenciés de protection, chacun devant être souple, ce que le législateur prit en compte en distinguant la sauvegarde de justice, la curatelle et la tutelle. Ce faisant - et c'est ici le point décisif - c'est le rôle thérapeutique des mesures qui est reconnu, comme l'explicita après-coup Jean Carbonnier :

«Il fallait arriver à une législation où à chaque malade sa dose de capacité pût être prescrite sur ordonnance du médecin. Car la psychiatrie moderne insiste sur cette espérance: une participation aux actes juridiques peut avoir des vertus de psychothérapie ${ }^{20}$.»

Mais personne ou presque n'évoque alors les modalités dans lesquelles ce travail thérapeutique spécifique relatif à la gestion des biens devra se faire. Koechlin en donne une explication qui souligne le paradoxe de la loi de 1968 :

«Les incidents qui ont conduit à vouloir modifier cette loi [de 1838] n'ont pas pour cause essentielle l'imperfection de celle-ci, mais en dernière analyse, ils ont tous pour motif une insuffisance de soins [...]. Par exemple, il est bien évident que dans une conception générale de la conception des soins psychiatriques, la manipulation thérapeutique de l'argent et des biens du malade y est intégrée ${ }^{21}$. »

La loi soutient ainsi la dissociation de la gestion des biens et celle de la personne alors qu'il est évident pour tout le monde, juristes comme psychiatres, que ces deux dimensions sont imbriquées et qu'elles relèvent du soin psychiatrique.

Ce paradoxe trouve une forme de résolution dans le déplacement du rôle donné aux médecins. Ce que la loi retire à l'institution asilaire est rendu sous une autre forme à leur responsabilité. Un examen médi-

19. Livre Blanc ..., op. cit.

20. Jean CARBONNIER, in Jacques MASSIP, op. cit. p. 23, « La loi de 1968 fut d'emblée conçue comme un instrument thérapeutique pour les médecins face aux états dits curables. »

21. Livre Blanc... op. cit, p. 394 
cal est rendu obligatoire pour qu'un juge puisse prononcer l'ouverture d'une mesure de protection au motif que le majeur est atteint d'une altération de ses facultés ${ }^{22}$. Ni les juristes ni les psychiatres n'entendent remettre en cause l'autorité médicale, bien au contraire. Mais pour les uns, cette autorité est d'abord celle d'une expertise fondée sur un savoir positif ${ }^{23}$. Pour les autres, elle est issue d'une pratique dont le sens réside tout entier dans sa performativité thérapeutique. Si cette divergence classique quant au rôle du médecin est bien connue des uns et des autres, nul n'a cependant intérêt à la clarifier. Habilement, une place a été laissée dans la procédure à un médecin «spécialiste » et à un médecin «traitant», dissimulant par ces qualifications évasives que le psychiatre incarne justement en une même personne le rôle d'expert et celui de thérapeute.

Cette nouvelle place des médecins laisse donc prise à une double critique :

- d'abord celle d'une médicalisation à outrance des mesures de protection, conduisant à une extension excessive des régimes d'incapacité «aux handicapés et aux vieillards ${ }^{24}$ ». Ce sera notamment le point de vue défendu par les sénateurs avant le vote de la loi. Cette critique entend donc plutôt limiter le savoir médical dans sa prétention à apporter des solutions thérapeutiques à des problèmes qui ne relèveraient pas de ce champ ;

- la place équivoque laissée aux médecins engendrera également une critique de «démédicalisation ${ }^{25}$ » présente dans quelques réserves formulées par les psychiatres au milieu des années 1960, ou plutôt dans leur crainte qu'elle ne tienne pas suffisamment compte de la nécessité d'inclure la gestion des biens dans une dynamique thérapeutique.

22. Article 490 du Code civil: "L'altération des facultés mentales ou corporelles doit être médicalement établie. » Cette obligation est légale et non pas laissée à l'appréciation souveraine du juge, qui est autorisé, par le Code de la procédure civile, à procéder «en toutes matières » à une expertise. Ce sont les sénateurs qui ont formulé cette proposition, les médecins psychiatres ne mettant alors pas en avant cette question de l'expertise.

23. «L'interrogatoire par le magistrat consistait en général à poser un certain nombre de questions puériles, à essayer de faire reconnaître à l'intéressé des pièces de monnaie, et à rechercher s'il avait une idée approximative de la consistance de ses biens. » Officieusement, il y avait des renseignements médicaux ; « le recours à la science médicale est officialisée ». cf. Bernard SALINGARDES, «La loi du 3 janvier 1968 », Revue pratique de psychologie de la vie sociale et d'hygiène mentale, $1968, \mathrm{n}^{\circ} 2, \mathrm{p} .80$.

24. «Rapport de M. Grand, pour la commission des affaires sociales devant le sénat », séance du 17 mai $1967, \mathrm{n}^{\circ} 111$, p. 370 et 372.

25. Erwan QUÉZÉDÉ, La protection des incapables majeurs. Son histoire et ses perspectives d'évolution, Thèse pour le diplôme d'Etat de docteur en médecine. Université d'Angers, 2003. p. 43. 
Ces deux critiques s'éteindront par le désintérêt d'une grande partie de la communauté médicale pour les questions tutélaires. Mais le paradoxe initial, consistant à dissocier protection des biens et de la personne alors que tous estiment que ces deux dimensions sont imbriquées, a rapidement des conséquences qui poseront des problèmes pratiques aux acteurs. En ne traitant pas de fond la question de la gestion de l'argent dans la prise en charge thérapeutique, et en acceptant l'équivoque de la procédure judiciaire d'incapacitation, les psychiatres se retrouveront coincés dans un entre-deux et n'investiront la protection des biens ni comme experts, ni comme thérapeutes. Ce faisant, c'est la démarche même de revitalisation de l'idéal psychiatrique par l'organisation sectorisée qui se trouve minée dès ses débuts. En acceptant, pour des raisons tactiques, la séparation institutionnelle de la protection de la personne et de la protection des biens proposée par la Chancellerie, les réformateurs de la psychiatrie d'après-guerre ne perdent pas seulement la main, ils se coupent également des moyens de mettre en œuvre la visée globale qui inspirait leur projet.

\section{Les motifs dits sociaux et la tutelle aux prestations sociales}

Ces transformations à venir sont difficiles à saisir en 1968. La psychiatrie règne encore sur la maladie mentale, et les recompositions que la notion de santé mentale parviendra tant bien que mal à thématiser ne sont pas encore bien identifiées. Paradoxalement, les brouillages futurs sont anticipés, mais certainement pour des raisons conservatrices. Le législateur prévoit en effet la possibilité d'ouvrir une mesure de protection pour des personnes dont le comportement expose à «tomber dans le besoin », que cela soit "par prodigalité, par intempérance, ou par oisivet $^{26}{ }^{26}$. Nul besoin alors de certificat médical. Le texte s'éloigne ici des considérations pré-successorales qui motivaient le conseil judiciaire pour se centrer directement sur les difficultés sociales engendrées par des dépenses inadaptées. Le public visé s'est ainsi clairement déplacé. Le Code civil prend ici une «orientation prolétaire ${ }^{27}$ » en se souciant d'assurer à chacun un minimum vital, ce que le rapporteur du projet de loi pressent :

«Toute une politique de défense familiale et sociale est ainsi peutêtre en germe dans ce texte ${ }^{28}$.

Ce tournant est encore mal appréhendé, aucune articulation n'étant alors prévue avec les tutelles aux prestations sociales ${ }^{29}$. Il conduira à

26. Article 489 du Code civil « $68 »$ » Il n'est pas possible d'ouvrir une mesure de tutelle pour des motifs dits sociaux. Seule une mesure de curatelle ou éventuellement une sauvegarde de justice peuvent être concernées.

27. Anne GOTMAN, Dilapidation et prodigalité, Paris, Nathan, 1995, p. 310

28. Rapport Pleven, op. cit. p.1323. 
introduire de nouveaux acteurs, les travailleurs sociaux, dans le dispositif de protection, et à ouvrir les mesures à des publics dont les biens sont peu importants.

La réforme de 1968 a marqué un tournant décisif dans l'organisation institutionnelle de la psychiatrie et du système de protection juridique. S'inscrivant dans la réforme des hôpitaux psychiatriques promue par les psychiatres réformateurs, la loi entendait réduire les effets ségrégateurs de l'asile et rompre avec l'exceptionnalité du droit qui l'organise. Peu nombreux sont alors ceux qui ont conscience qu'elle consacre l'émancipation de l'activité de gestion de biens des malades du travail psychiatrique. Elle redéfinit un nouvel équilibre entre le pouvoir du médecin, celui du juge et celui des familles.

\section{UNE MISE EN GUUVE SOUPLE ET L'ARRIVEE DES TRAVAILLEURS SOCIAUX}

La loi du 3 janvier 1968 a donc réorganisé les prérogatives de la psychiatrie et celles de la justice civile, tout en donnant au nouveau dispositif de protection une grande souplesse dans son application. Cette souplesse se traduit par les différentes modalités d'organisation qui s'offrent au juge des tutelles, s'exprimant aussi bien en termes de graduation des pouvoirs donnés au mandataire en charge de la protection que du statut même des mandataires. La loi prévoit alors la possibilité d'ouvrir une tutelle complète, une administration légale sous contrôle judiciaire, ou une gérance de tutelle; elle donne également un statut spécifique à certains membres de la famille, à des personnes morales, à des établissements hospitaliers ou encore à l'État. En fonction de la combinaison choisie par le juge, le type d'acteur amené à prendre un pouvoir sur les personnes à protéger varie. Nous examinons maintenant comment le dispositif prévu en 1968 sera appliqué et analysons le rôle important pris par trois types d'acteurs, la famille, les professionnels de l'action sociale, et les établissements hospitaliers.

\section{Un héritage du passé : la gérance de tutelle}

Au lendemain de la réforme de 1968, la modalité la plus pratique pour faire transition entre l'ancien et le nouveau cadre juridique est,

29. Vingt-cinq ans plus tard, au détour d'une note, Jacques Massip expliquera les raisons du manque d'articulation entre les deux mesures. Le Garde des Sceaux aurait, selon l'ancien secrétaire de Carbonnier, voulu fusionner les deux tutelles mais il s'est heurté à l'opposition du ministère des Affaires sociales, car il se méfiait - et se méfie toujours - des tutelles familiales et leur préfère les tutelles administratives, exercées par des organes sociaux ou parasociaux qu'il contrôle. Cass. $1^{\text {ère }}$ civ. 27 janvier 1993, Gazette du Palais, 1-2 déc 1993, note J. Massip. 
dans une grande majorité des cas, celle de la gérance en établissement :

«Si, eu égard à la consistance des biens à gérer, le juge des tutelles constate l'inutilité de la constitution complète d'une tutelle, il peut se borner à désigner comme gérant de la tutelle [...] un préposé appartenant au personnel administratif de l'établissement de traitement $^{30}$.»

Derrière la nouveauté se dissimule en fait la reconversion du régime d'administration provisoire des biens qui était gérée dans les asiles par des préposés d'établissement : la protection de la personne est toujours dévolue à l'équipe médicale alors que l'agent administratif qui gère les biens ne peut aucunement prendre des décisions au regard de la personne du protégé.

Pour certains, la continuité avec le régime d'administration provisoire est pratique et rassurante. Pour d'autres au contraire, elle ne respecte pas l'esprit de la réforme de 1968 dont l'un des nœuds est la dissociation des mesures thérapeutiques et des mesures de protection des biens : "Cette exception ne peut être le cheval de Troie de la loi du 3 janvier 1968. » Ces mesures de gérance devraient être considérées comme exceptionnelles.

La mesure est en effet souple et peut être aussi bien prise en charge par des préposés d'établissement pour les personnes hébergées en institution, que par des administrateurs indépendants, laissant craindre qu'elle se généralise et qu'elle entérine une véritable tutelle du pauvre. L'article 499 a en effet officialisé ce que la pratique avait déjà généralisé. Cette mesure est destinée aux cas où les biens de la personne à protéger ne sont pas importants.

Si le risque de généralisation d'une véritable « tutelle du pauvre » a été bien anticipé par les commentateurs ${ }^{31}$, sa localisation est mal identifiée. Les modalités de financement qui seront prévues quelques années plus tard ne permettront pas aux gérants de tutelle d'être financés quand les revenus du protégé sont très faibles. La «tutelle du pauvre » ne passera pas tant par cette forme allégée que par le développement d'une organisation spécifique des tutelles d'État à travers l'activité professionnelle de protection, sur laquelle nous reviendrons un peu plus loin. Surtout, la gérance de tutelle ne sera jamais véritablement

30. Article 499 du Code civil « $68 »$.

31. C. GEFFROY et N. BELLEC, « La tutelle d'État, Analyse du décret du 6 novembre 1974 », Perspectives psychiatriques, décembre 1975. La formule est visiblement utilisée dans les débats entre professionnels. En soulignant que cette gérance n'est pas une véritable mesure de tutelle, les juristes répondent à l'avance à la critique d'une mesure qui ne serait qu'une reproduction de l'ancien dispositif d'administration provisoire. 
adaptée aux évolutions de la politique psychiatrique. Au milieu des années 1960, certains psychiatres avaient imaginé des modes d'organisation de la protection tutélaire qui seraient analogues à ceux de la psychiatrie, ne se référant plus tant à une logique d'établissement qu'à une logique territoriale ${ }^{32}$. Cette volonté d'articulation de l'administration des biens et de l'organisation psychiatrique disparait ensuite. Au moment où la politique de sectorisation est véritablement prise en compte dans le statut des établissements hospitaliers en $1985^{33}$, l'enjeu de l'adaptation de la gérance des biens à la nouvelle organisation des soins n'est pas posé. Il ne le sera pas davantage au moment de la loi du 27 juin 1990 sur les hospitalisations sans consentement, cette indifférence étant par exemple illustrée par l'absence de lien entre le curateur à la personne, prévu dans les établissements psychiatriques, et l'activité de protection de la personne des curateurs d'État.

\section{Le recours à l'administration légale et aux mandats familiaux}

La facilitation du recours aux familles prévue par la loi de 1968 connaît rapidement un grand succès. Il faut cependant préciser que la loi avait été ambivalente au regard de cet acteur «particulier», considéré bien souvent comme « pourvoyeur naturel de la protection. » Elle a donné une priorité non vraiment explicitée à la désignation d'un membre de la famille, résidant uniquement dans l'ordre des articles de $\operatorname{loi}^{34}$. Cette ambivalence s'explique par la prise en compte par le législateur de deux tendances contrastées.

D'abord, il faut prendre en compte la place importante prise par la famille restreinte, dite parfois conjugale. La possibilité est ouverte de désigner un membre de la famille comme mandataire sans avoir recours au conseil de famille ${ }^{35}$. Le conseil de famille n'est plus obligatoire et il ne sera de fait presque plus utilisé. Ce changement résulte du «souci de faire davantage confiance aux proches» et de simplifier l'exercice des mesures. L'administration légale sous contrôle judi-

32. «L'appareil chargé de la protection des biens ne peut plus être accroché à l'institution, mais doit avoir un ressort territorial. Cet organisme doit être proche et accessible. L'utilité d'une protection des biens ne se fait pas sentir pour tous les malades. » Hubert MIGNOT, Livre blanc de la psychiatrie française, t.1, 1965, p.113.

33. Ce point permet en partie d'expliquer pourquoi une organisation sectorielle de la gérance de tutelle n'a pas été véritablement discutée à la fin des années 1960 et au début des années 1970.

34. Les deux modes d'exercice familial de la tutelle étant placés avant tous les autres. Michel BAUER, Thierry FOSSIER, Laurence PÉCAUT-RIVOLIER, La réforme des tutelles. Ombre et Lumière, Paris, Dalloz, 2006, p. 118.

35. L'organisation d'une tutelle incomplète donnant les pouvoirs d'un administrateur légal au membre de la famille nommé va rencontrer un succès très important. 
ciaire n'implique en effet aucune obligation familiale autre que celle qui pèse sur le membre de la famille choisi par le juge.

La seconde tendance se caractérise plutôt au contraire par une défiance vis-à-vis de la famille. Le rapport Pleven est explicite à ce sujet :

«Il faut souligner que dans bien des cas, les troubles mentaux ont, plus ou moins, pour origine des conflits de caractère familial ou conjugal. Il est bien certain que, dans de telles hypothèses, le rôle à tenir par la famille doit être envisagé avec beaucoup de circonspection. En tout état de cause, ce n'est jamais à la famille qu'il peut être fait appel pour apprécier l'état d'un malade, l'étendue de son incapacité à gérer lui-même ses affaires, la nature des mesures de protection dont il convient de l'entourer ${ }^{36}$.»

Cette tendance se traduit concrètement par l'obligation faite au juge de tenir compte d'un constat médical au moment de juger de l'état de la personne. Anne Gotman considère que « la famille a fait les frais » de la nouvelle alliance entre les juges et les médecins.

Le succès rencontré par l'administration légale confiée à un membre de la famille conduit plutôt à constater que la famille ne désigne plus la même entité. Alors qu'elle était étendue et s'incarnait sous la forme du conseil de famille jusqu'en 1968, elle s'est réduite le plus souvent depuis lors au proche qui est directement en charge du mandat de protection.

\section{L'émergence de l'activité professionnelle de protection}

Parallèlement à la solution familiale et à la gérance hospitalière, s'est développée une nouvelle modalité d'organisation professionnelle de l'activité tutélaire. Cette activité a répondu aux difficultés rencontrées par les juges des tutelles quand aucun membre de la famille n'est jugé apte à exercer le mandat de protection, quand les ressources de la personne ne permettent pas d'assurer le financement de l'activité d'un gérant privé de tutelle et quand une gérance par un établissement de soin n'est pas possible. Ce cas de figure n'a pas véritablement été planifié en 1968. Le législateur a cependant rendu possible la nomination de l'État, à travers ses services déconcentrés ${ }^{37}$, comme mandataire d'une protection, ou d'une personne morale. L'organisation de l'activité professionnelle de protection au sein d'associations agréées par les services de l'État imbrique ces deux possibilités légales. À la

36. Rapport Pleven, op. cit., p. 1313.

37. Le décret de 1974 prévoit que les DDASS peuvent être directement sollicitées par les juges des tutelles. Elles vont donc s'organiser pour développer cette activité non spécifiquement financée. Certaines administrations départementales vont mandater quelques agents en interne. 
fin des années 1970, la plupart des DDASS externalisent le mandat de protection qui leur est confié en le déléguant aux associations, notamment familiales, qui sont reconnues par leur habilitation à gérer des tutelles aux prestations sociales ${ }^{38}$. La proximité entre l'activité de gestion des tutelles aux prestations sociales et le mandat pour exercer des tutelles d'État est à ce moment-là déterminante. Au-delà de la proximité lexicale, ce sont en effet les mêmes autorités, en la personne des juges des tutelles, qui sont amenées à prononcer les deux types de mesure $^{39}$. La création d'une nouvelle prestation sociale, l'Allocation adulte handicapée (AAH) scelle le rapprochement de ces deux activités. De nombreuses personnes considérées comme incapables de protéger leur intérêt peuvent bénéficier d'une AAH. «Servie ${ }^{40}$ comme une prestation familiale, celle-ci peut être mise sous tutelle sociale. La juxtaposition d'une mesure civile, justifiée par le constat médical de l'altération des facultés personnelles, et d'une mesure aux prestations sociales, justifiée par le repérage d'une gestion défectueuse de ces prestations, permet de financer une mesure civile par une tutelle aux prestations sociales. Les répercussions de ce rapprochement ne concernent pas seulement le financement des mesures mais aussi et surtout la nature même de l'activité de protection. Les associations mandatées délèguent le plus souvent l'activité de protection à des professionnels de l'action sociale qui ne limitent pas leur intervention des biens mais entendent également «accompagner la personne ${ }^{41}$.»

Si elle constitue une réponse pratique aux difficultés des acteurs, cette arrivée des travailleurs sociaux dans le domaine de l'exercice des mesures de protection est, dans premier temps, freinée par les services de l'État. Si les réticences sont d'abord financières, l'argument officiellement présenté relève de l'organisation socio-civile de la solidarité :

38. Il faudra attendre 1983 pour qu'une ligne budgétaire spécifique dans les crédits de l'action sociale soit créée. Cette nouvelle orientation organisationnelle répond visiblement à des attentes très fortes, à en croire la multiplication du nombre de mesures et d'associations juste après cette circulaire. Le nombre de mesures d'État croît de $60 \%$ en 1984, passant d'un peu plus de 40000 à 70000 mesures. Le nombre d'associations triple entre 1983 et 1985, passant de 44 à 115.

39. Le cadre juridique des tutelles aux prestations sociales a été défini par une loi de 1966 intégrée alors au Code de la sécurité sociale. Elle autorise un juge d'instance à mettre sous tutelle différentes prestations sociales, initialement plutôt des allocations familiales, si celles-ci ne sont pas utilisées pour l'amélioration des conditions de vie des bénéficiaires.

40. Art. 37 de la loi d'Orientation du 30 juin 1975.

41. Dans un projet de code de déontologie prévu pour les «intervenants tutélaires », il est prévu que l'intervenant soit chargé «d'accompagner et d'assister la personne dans ses besoins psychiques, psychologiques et physiques; de chercher à développer dans le cadre de son mandat les potentialités de la personne dans tous les domaines de la vie sociale. ", Bulletin ANDP, 04 mai 1989. 
«Pourquoi une tutelle professionnelle? Ne risque-t-elle pas d'accroître la désagrégation des solidarités naturelles et notamment familiales $?^{42} \gg$

Finalement, à la fin des années 1980, un compromis se noue, à la suite de quelques décisions judiciaires, entre les services de l'État et les associations tutélaires conduisant à financer les mesures civiles de protection sans que soit directement reconnue la professionnalité des délégués intervenant dans les associations tutélaires ${ }^{43}$.

Près de vingt ans après la réforme de 1968, l'organisation de la protection tutélaire s'est donc stabilisée. Une solution technique s'est pérennisée dans les établissements de soin sans qu'aucune articulation avec les thérapeutiques psychiatriques ne soit organisée ; le rôle souvent informel des familles a été massivement légalisé avec le développement de l'administration légale sous contrôle judiciaire, celles-ci apparaissant alors comme « pourvoyeurs naturels des soins »; de nouveaux acteurs sont enfin apparus avec l'organisation d'une activité professionnelle de protection laissant une place importante aux techniques d'intervention sociale, et revendiquant «l'accompagnement de la personne ».

\section{LES FONDEMENTS ET LES OBJECTIFS DE LA REFORME DE 2007}

Rapidement cependant, la stabilisation pratique de ce dispositif pluriel se révèle insuffisante. Dès le début des années1990, les juges des tutelles se mobilisent via la toute jeune Association nationale des juges d'instance, créée notamment par Thierry Fossier, groupe qui se réunit alors autour de Jacques Massip afin de proposer un projet de réforme à la Chancellerie et préciser ce qui est attendu juridiquement par «protection de la personne ». Au milieu des années 1990, les administrations centrales s'inquiètent de l'augmentation considérable du nombre

42. Compte-rendu de la réunion de juin 1985 réunissant les ministères de tutelles et les représentants associatifs et syndicaux, Bulletin ANDP, 04-1985.

43. En moins d'un an, plusieurs décisions se succèdent qui permettent de nouer un compromis a minima sur le problème de la définition de l'activité professionnelle de protection, décisions concernant aussi bien l'organisation des mesures que leur portée et leur financement : la curatelle d'État est officialisée par décret n 88-762 du 17 juin 1988, ce qui rend possible son financement ; le 18 avril 1989, la cour de Cassation entérine la légalité du financement indirect de mesures civiles par des mesures de tutelle aux prestations sociales en justifiant le refus de mesures qui ne sont pas financées par les associations et les demandes d'ouverture de TPSA accompagnant les mesures civiles; le même jour, la cour prononce un arrêt relatif au contenu du mandat donné aux tuteurs: «Les régimes civils d'incapacités ont pour objet d'une manière générale de pourvoir à la protection de la personne et des biens de l'incapable.» 
de mesures ${ }^{44}$ et des rapports sont commandités. C'est en 1999 qu'une alliance se noue entre juristes et associations tutélaires, sous l'impulsion notamment de l'Union nationale des associations familiales (UNAF), qui a commandité à Thierry Fossier la formulation d'un avant-projet. Il faudra encore quelques années et la sollicitation du médiateur de la République et du Conseil économique et social pour que la loi soit proposée au Parlement, et que les inquiétudes provenant des financeurs - que ce soient les conseils généraux ou le ministère des Affaires sociales - soient en partie levées.

Nous examinons plus précisément maintenant trois des principaux axes qui structurent la loi de 2007 et qui ont été pour la plupart formulés dès le rapport Favard : la reformulation de la priorité familiale ; un meilleur ciblage des publics en réservant les mesures de protection aux personnes ayant une «altération des facultés personnelles»; l'extension de l'activité de protection à la protection de la personne.

\section{La reformulation de la priorité familiale et la dissociation implicite des mandats sociaux et familiaux}

Dans les motifs du projet de loi de la réforme votée en mars 2007, le législateur affirme fortement son souhait de privilégier les tutelles familiales :

"Cette protection doit alors être envisagée d'abord au sein du groupe familial, protecteur naturel du majeur vulnérable, avant toute désignation d'un tiers ${ }^{45}$. »

Ce souhait formulé tient peu compte des difficultés que rencontrent les familles dans certaines situations pour assurer cette protection. On peut dire que la dimension performative des motivations du législateur se confronte à un contexte social peu malléable ${ }^{46}$. L'introduction d'une nouvelle mesure - le mandat de protection future - apparait en revanche comme très importante dans l'économie générale du dispositif

44. La nécessité d'une réforme s'est fait sentir dès le milieu des années 1990, notamment en raison de l'explosion du nombre de mesures. En 10 ans, entre 1990 et 2000, l'augmentation du nombre d'ouverture de mesures est de $30 \%$, passant de 40000 en 1989 à 57000 mesures annuelles en 1999. Cette augmentation est beaucoup plus importante encore concernant les mesures d'État, la curatelle d'État, tout juste officialisée, connaissant la hausse la plus spectaculaire, le nombre de mesures ouvertes ayant quasiment été multipliées par cinq entre 1990 ( $\mathrm{N}=2$ 567) et 2002 ( $\mathrm{N}=11$ 979). Sources, Répertoire civil, Cellule étude et recherches de la direction des affaires civiles et du sceau.

45. Projet de loi $n^{\circ} 3462$ portant réforme de la protection des majeurs, présenté à l'Assemblée nationale par Pascal Clément, garde des Sceaux. Enregistré à la présidence de l'Assemblée nationale le 28 novembre 2006, p. 4.

46. Les travaux de sociologie de la famille soulignent la forte recomposition des solidarités familiales et que les juges prennent acte pour la plupart de situations sociobiographiques hétérogènes. 
de protection. L'hypothèse peut être faite que les mandats familiaux jusqu'ici décidés par le juge seront à l'avenir en grande partie décidés de manière anticipée par la personne elle-même grâce au mandat de protection future. Cette transformation est importante car elle entérine en fait un nouveau régime d'incapacités déjudiciarisé au maximum. Ce faisant, une véritable rupture est opérée dans l'économie générale du dispositif de protection des incapacités. Un nouveau droit commun s'annonce autour de ce principe de prévisibilité, soulignant la spécificité des mesures qui n'auront pas été organisées à l'avance et consolidant le statut particulier réservé aux majeurs pour qui l'obligation de protection est prononcée judiciairement.

Pour ces derniers, le recours à l'activité professionnelle de protection sera probablement le plus souvent mis en œuvre. La loi organise donc les modalités d'application des principes retenus :

«Afin de mieux encadrer l'organisation et le fonctionnement du secteur et d'intégrer la dimension sociale de l'intervention tutélaire, il est proposé d'inscrire cette activité dans le champ de l'action sociale ${ }^{47}$. »

Les règles et les principes de l'activité sont donc définis par le Code de l'action sociale et des familles, notamment suite à la loi de mars 2002. Les associations sont dorénavant considérées comme des « services mandataires judiciaires à la protection des majeurs ». Leur activité est soumise à l'habilitation par la préfecture et est planifiée dans le cadre des «schémas d'organisation sociale et médicosociale ». Un serment doit être prêté et différents documents prévus par la loi de 2002 sont adaptés : une notice d'information, une charte des droits et des libertés de la personne protégée, un document individuel de protection du majeur (DIPM) sont mis en place. Ce faisant, l'activité de protection passe très nettement du côté des compétences du travail social :

"Le travail social, notamment en réseau, est ici mis à l'épreuve. Il est, décidément, le pilier, sinon du texte nouveau, en tout cas de sa réussite ${ }^{48}$.»

L'officialisation de l'activité professionnelle de protection s'inscrit logiquement à la suite de la mise en œuvre du droit tutélaire depuis une trentaine d'années. Ce faisant, elle spécifie cependant un régime socio-civil spécifique de protection qui renforce la différence entre les mandats ordinaires de droit civil et des mandats qui s'inscrivent dans les règles particulières de l'action sociale. Elle participe au souci de professionnalisation des intervenants et s'inscrit dans la logique d'une évolution de l'État social qui s'appuie fortement sur le droit de l'action

47. Projet de loi 2006, exposé des motifs, op. cit., p. 7.

48. Thierry FOSSIER, op. cit., 2007, p. 680. 
sociale. Il faut cependant noter qu'aucun lien n'a été établi explicitement avec les mesures prévues par la loi du 11 février 2005 pour l'égalité des droits et des chances, la participation et la citoyenneté des personnes handicapées, ce qui traduit certainement la difficulté à positionner ces régimes socio-civils de protection qui relèvent aussi bien du droit civil que du droit social.

\section{Le recentrement sur l'altération des facultés mentales}

La seconde orientation axée sur le statut spécifique réservé aux personnes protégées juridiquement par des professionnels se caractérise par la distinction entre les mesures d'action sociale et éducative et les mesures civiles, distinction censée répondre au problème posé par l'augmentation conjointe des phénomènes de précarisation et des problèmes de santé mentale. Cette orientation répond à ce que le rapport des trois inspections considérait comme une "dérive », et que le rapport Favard qualifiait d' "utilisation devenue abusive de mesures de protection des majeurs lorsqu'elles pallient les insuffisances des dispositifs d'accompagnement social ${ }^{49}$. » À leur suite, les motifs de la loi formulent cette critique au dispositif :

«Sous l'influence d'une évolution socio-économique marquée par le vieillissement de la population et l'importance des phénomènes de précarité et d'exclusion, de nombreuses mesures sont prononcées pour des raisons plus sociales que juridiques [...]. La réforme est donc dictée par le souci de recentrer le dispositif de protection juridique sur les personnes réellement atteintes d'une altération de leurs facultés personnelles ${ }^{50}$.»

Avec la loi du 5 mars 2007, l'altération devient le seul critère permettant d'établir « une ligne de partage » entre les publics, même si elle n'est pas accompagnée d'une réforme de l'évaluation du besoin de protection. Les motifs dits sociaux, qui jusqu'alors pouvaient justifier l'ouverture d'une mesure de protection, sont supprimés du Code civil alors qu'une nouvelle mesure est créée, la mesure d'accompagnement social personnalisé, destinée, comme le dit Laurence MaugerVielpeau, aux «nouveaux prodigues ${ }^{51} »$. Cette distinction doit permettre de ne plus confondre les personnes relevant de l'action sociale et celles relevant d'une décision judiciaire. Cette volonté de distinguer les motifs sociaux des véritables motifs, qui seraient médicaux, se confronte cependant à la difficulté de dissocier l'imbrication de ces deux dimensions dans de très nombreuses situations. Elle renvoie par

49. Rapport FAVARD (dir.), ministère de la Justice, avril 2000, p. 3.

50. Exposé des motifs du projet de loi, p. 3-4.

51. Laurence MAUGER-VIELPEAU, «Les destinataires de la loi $n^{\circ} 2007-308$ du 5 mars 2007 : une loi d'action sociale ?», Revue de droit sanitaire et social, $\mathrm{n}^{\circ} 4,809-812$, juillet-août 2007, p. 811. 
ailleurs au rôle des mesures de protection pour les personnes atteintes de troubles psychiques, problème qui n'a cependant que très peu été discuté au moment de la réforme. Cette difficulté avait pourtant été soulevée avec une certaine insistance par les associations au début des années 1990, confrontée notamment parfois à des problèmes de violence $^{52}$. Les rapports commandés par le ministère ont évité pour une bonne part le sujet. De manière emblématique, aucun psychiatre ou représentant de la psychiatrie n'a fait partie du groupe de travail constitué autour de Jean Favard. Les difficultés spécifiques posées par le public que le rapport nomme «handicapés psychiques » ou «psychotiques » sont identifiées ${ }^{53}$, mais pour être rejetées à l'extérieur des préoccupations du groupe de travail. Faute d'être véritablement ouvert avec les acteurs de la psychiatrie, ce sont des consignes sécuritaires qui ont été proposées la plupart du temps : suppression de l'argent liquide lors de la remise du budget ; obligation de la présence de deux personnes lors de la réception du public dans une délégation; mise en place de portiers de rue (gâche électrique et interphone).

La non-prise en compte des difficultés spécifiques posées par les publics touchés par une maladie mentale est une forme d'aveu de l'impossibilité de penser directement l'articulation des décisions judiciaires, des pratiques d'accompagnement social, et du travail thérapeutique ; il révèle la difficulté de donner un contenu à l'activité de protection des biens et de la personne ; enfin, et surtout, il rend visible le malaise relatif à la définition des publics visés par les mesures de protection.

\section{L'organisation de la protection de la personne}

Cette absence d'articulation avec les acteurs de la psychiatrie est d'autant plus paradoxale que les mandataires à la protection voient leurs prérogatives s'étendre à la protection de la personne.

Avec la reconnaissance législative de ce mandat, c'est très clairement le combat mené depuis plus de vingt ans par de nombreux juges des tutelles au côté des associations qui se trouve couronné ${ }^{54}$. La ques-

52. Un drame a même alerté les différentes institutions. En 1996, une déléguée à la tutelle est tuée par un majeur protégé. Certaines associations tutélaires réclament alors que soit davantage prise en compte la différence entre les publics et l'UNAPEI réclame de pouvoir «refuser d'assurer la protection juridique de personnes malades mentales. »Cette demande rencontre l'hostilité des magistrats. Le président de l'Association nationale des juges d'instances interpelle les pouvoirs publics et réaffirme la nécessité d'associations généralistes. Cf. Tutelle Info $\mathrm{n}^{\circ} 83$ et $\mathrm{n}^{\circ} 90$.

53. Rapport Favard, p. 23

54. La littérature juridique qui a été consultée laisse cependant peu de place à ce débat dans lequel Claude Lombois a notamment défendu «des positions civilistes » réfractaires à donner un pouvoir au mandataire pour des actes relevant de la vie privée. 
tion de la protection de la personne n'est alors plus considérée comme le monopole des psychiatres, mais est traitée directement par les civilistes. Après l'arrêt rendu le 18 avril 1989, la réforme de 2007 confirme cette logique et transforme le principe général de la protection des majeurs en opérant un double mouvement.

D'un côté en effet, la protection de la personne consiste à préserver, voire élargir ses capacités «naturelles » malgré l'incapacité « de droit » qui la frappe :

«En matière personnelle, le principe devrait être de la primauté du consentement de la personne et de la subsidiarité de l'incapacité, principe qu'une réforme pourrait heureusement préciser ${ }^{55}$. 》

C'est donc une véritable limitation de la présomption d'incapacité, en ce qu'elle a de continu, qui est énoncée. Ceci a une double conséquence. D'une part, les réévaluations régulières de l'incapacité présumée de la personne sont rendues obligatoires ${ }^{56}$; d'autre part, le consentement est recherché dans des domaines où, jusqu'à présent, il ne l'était pas ${ }^{57}$. Ce premier mouvement consiste donc à considérer le principe de «protection de la personne » comme une extension de ses capacités à agir et à consentir.

D'un autre côté, la protection s'actualise au travers d'un contrôle plus étendu donné au mandataire chargé de veiller à la sécurité de la personne vulnérable. Lorsque le principe général ne peut s'appliquer, notamment parce que la personne sous protection ne peut exprimer sa volonté, le législateur prévoit cette fois-ci une extension du pouvoir octroyé à un tiers en matière de tutelle.

La réforme de 2007 est de ce point de vue catégorique, même si la formulation du principe est entourée de très nombreuses précautions et limites à son application. L'article 459 , dont le premier alinéa déjà cité entend encadrer l'intervention du mandataire, continue comme suit :

«Dans tous les cas, la personne chargée de la protection du majeur peut prendre à l'égard de celui-ci les mesures de protection rendues nécessaires par le danger auquel il s'exposerait, du fait de son comportement. »

Ainsi, le mandataire peut agir sur la personne du majeur à l'encontre de son consentement, non seulement en matière patrimo-

55. Jean HAUSER, article 878, Revue trimestrielle de droit civil, 1996.

56. Article 7 de la loi révisant le chapitre 11 , titre, $1^{\mathrm{er}}$ du Code civil. Article $\mathrm{n}^{\circ} 441 \mathrm{du}$ Code civil : «Le juge fixe la durée de la mesure sans que celle-ci puisse excéder cinq ans. »

57. Article 459 du Code civil : "La personne protégée prend seule les décisions relatives à sa personne dans la mesure où son état le permet. » 
niale, mais aussi «en matière personnelle». Le pouvoir octroyé au mandataire est élargi en même temps qu'il est davantage soumis au contrôle du juge.

En donnant un pouvoir de protection de la personne à des acteurs qui ne sont pas des thérapeutes, la loi prend de fait en compte une acception étendue des pratiques de « soin ».

\section{CONCLUSION}

Le parcours mené d'une réforme à l'autre peut maintenant être récapitulé au regard des trois influences qui se sont exercées sur l'évolution du droit tutélaire.

La première influence prise en compte a été celle de l'évolution de la politique psychiatrique. Il a été montré comment le savoir et l'activité psychiatriques ont été à un moment donné centraux dans la mise en œuvre du droit des incapacités, puis comment ils ont été progressivement relégués, que ce soit par un certain dessaisissement des acteurs de la psychiatrie ou par une évolution de leur place. Les rôles de thérapeute et d'expert, tenus ordinairement par le psychiatre, ont été dissociés. Les conséquences de cette dissociation semblent ne pas avoir fait l'objet de beaucoup de débats dans la genèse de la réforme de 2007 et sont peu explicites. Deux questions restent notamment en suspens : celle de l'articulation de la gestion des biens et de la dimension thérapeutique de la protection de la personne, d'une part ; celle du rôle du corps médical dans l'amalgame dénoncé par les rapports administratifs et repris par le projet de loi entre les motifs médicaux et les motifs sociaux, d'autre part.

La seconde influence prise en compte a été celle des évolutions de l'État social. Le droit des incapacités et de leur protection n'est devenu que sur le tard une ressource dans la gestion de la question sociale. Pendant longtemps, la question de la capacité civile des personnes pauvres ne s'est pas posée parce que les maigres biens qu'ils avaient à gérer n'étaient pas pris en considération ou mis sous d'autres formes de dépendance. Cette question est revenue sur le devant de la scène en raison de la transformation de la nature des biens des plus démunis. Les biens à protéger ne se sont plus seulement constitués des héritages familiaux, mais également des revenus du travail et de la solidarité. Entre le modèle dominant de la propriété privée qui servit de base aux régimes de protection en 1804 puis en 1838, et les sources de revenus qu'il s'agit de protéger pour les majeurs non internés, la nature des biens a considérablement évolué : salaires, revenus indirects liés au travail, retraite, assurance chômage, pension d'invalidité, mais aussi prestations sociales doivent maintenant être aussi protégés. La diffu- 
sion massive de ces nouvelles sources de revenus est parallèle à l'émergence de nouveaux droits, qualifiés par beaucoup de droits sociaux ; elle est venue complètement transformer le rôle des mesures tutélaires. Il ne s'agit plus seulement d'interdire des personnes civilement majeures afin qu'elles ne dilapident pas le patrimoine familial. Il s'agit également de permettre à certaines personnes de bénéficier de prestations auxquelles elles ont droit et de surveiller par la même occasion l'usage qu'elles en font. L'émergence d'une nouvelle activité et l'instauration d'un régime socio-civil d'incapacités-protection révèlent cependant le malaise de l'État sur la « question sociale».

La troisième influence prise en compte a été celle des évolutions dans la figuration civile de la personne. Le droit des incapacités n'a pas été exempt de la préoccupation qui s'est diffusée dans le droit de la famille, dans celui de la santé, et dans les institutions sanitaires et sociales dans une période récente, et qui donne au consentement de la personne une place toujours plus grande dans les activités de soin et de protection. La formalisation de la «protection de la personne » illustre particulièrement ce souci de préserver le plus possible la reconnaissance de l'autorité du consentement des individus concrets tout en autorisant de manière encadrée des tiers à intervenir à la place de la personne dans des cas spécifiques.

Plus largement, l'évolution du droit tutélaire met en exergue la tension entre le souci de «déspécifier» les régimes de protection et celui de limiter leur application à des publics-cibles. Une double interprétation de la réforme de 2007 est possible. La graduation de différents types de mesure peut être considérée comme participant d'une « déspécification » des mesures de protection et conduisant à une normalisation de la prise en compte des multiples formes de vulnérabilité. Inversement, la séparation entre les mesures conventionnelles et les mesures contraintes peut conduire à interpréter la réforme comme une « respécification» du droit des incapacités. Autrement dit, c'est bien un nouvel équilibre entre droit commun et droit d'exception que la loi du 5 mars 2007 a cherché à instaurer. 